\title{
Genomics of Myeloproliferative Neoplasms
}

\author{
Katerina Zoi ${ }^{1}$ and Nicholas C.P. Cross ${ }^{2,3}$
}

1. Haematology Research Laboratory, Biomedical Research Foundation, Academy of Athens, Athens, Greece

2. Wessex Regional Genetics Laboratory, Salisbury District Hospital, Salisbury, UK

3. Faculty of Medicine, University of Southampton, Southampton, UK

Corresponding author:

Professor Nicholas C.P. Cross

Wessex Regional Genetics Laboratory

Salisbury District Hospital

Salisbury SP2 8BJ, UK

Tel +44 1722429080

Fax +44 1722331531

Email: ncpc@soton.ac.uk 


\begin{abstract}
Myeloproliferative neoplasms (MPN) are a group of related clonal hematological disorders characterized by excess accumulation of one or more myeloid cell lineages and a tendency to transform to acute myeloid leukemia. Deregulated JAK2 signaling has emerged as the central phenotypic driver of $B C R-A B L 1$-negative MPN and a unifying therapeutic target. In addition, MPN show unexpected layers of genetic complexity, with multiple abnormalities associated with disease progression, interactions between inherited factors and phenotype driver mutations, and effects related to the order in which mutations are acquired. Although morphology and clinical laboratory analysis continues to play an important role in defining these conditions, genomic analysis is providing a platform for better disease definition, more accurate diagnosis, direction of therapy and refined prognostication. There is an emerging consensus with regard to many prognostic factors, but a clear need to synthesize genomic findings into robust, clinically actionable and widely accepted scoring systems, and well as the need to standardize the laboratory methodologies that are employed.
\end{abstract}




\section{Introduction}

The World Health Organization classification ${ }^{1}$ recognizes seven subtypes of myeloproliferative neoplasms (MPN). Chronic myeloid leukemia is unique in that is defined by the presence of a specific somatic abnormality, the $B C R-A B L 1$ fusion gene. Of the $B C R-A B L 1$-negative MPN (the focus of this review), polycythemia vera (PV), essential thrombocythemia (ET) and primary myelofibrosis (PMF) are often referred to collectively as classic MPN. Chronic neutrophilic leukemia (CNL), chronic eosinophilic leukemia (CEL) and MPN unclassifiable (MPN-U) are much less common and referred to as atypical or non-classic MPN. During their diagnostic work up, MPN need to be carefully distinguished from other myeloid entities by a combination of morphologic, clinical laboratory, cytogenetic, molecular genetic and, increasingly, genomic analysis.

\section{Classic BCR-ABL1 negative MPN}

\section{Deregulation of JAK2/STAT signalling}

Direct or indirect dysregulation of JAK2 signalling by somatically acquired mutations has emerged as a central phenotypic driver of classic MPN. JAK2 is a non-receptor tyrosine kinase which plays an essential role in transducing signals from class 1 cytokine receptors critical for normal myelopoiesis, notably the erythropoietin receptor, the thrombopoietin receptor (TPOR; encoded by the MPL gene) and the granulocyte colony-stimulating factor receptor. ${ }^{2,3}$

The principal driver mutations in MPN are indicated on Figure 1. JAK2 is directly activated by acquisition of the JAK2 V617F mutation, seen in the majority of MPN. ${ }^{4-7}$ JAK2 V617F mutation burdens are usually higher in PV and PMF compared to ET, with many cases demonstrating a homozygous mutant clone. ${ }^{8}$ Mutations of JAK2 exon 12 , typically complex insertion/deletion events, are seen in roughly one third of cases of JAK2 V617F-negative PV. ${ }^{9}$ 
Indirect dysregulation of JAK2 signalling occurs principally by activating mutations in MPL or CALR. MPL mutations are located in exon 10 and most commonly result in amino acid substitutions at W515 of the thrombopoietin receptor (TPOR). ${ }^{10,11}$ W515 is located within a small amphipathic motif of the human receptor at the junction between the transmembrane and cytoplasmic domains that is required for maintaining TPOR in its inactive conformation and prevents its autonomous activation; mutation of this residue abrogates this function thereby constitutively activating JAK2 and downstream signalling. ${ }^{10,12}$

CALR exon 9 mutations are found in about $25-30 \%$ of patients with ET and PMF. ${ }^{13,14}$ All pathogenic CALR result in a common frameshift encoding a novel C-terminus with a high positive charge, with $80 \%$ of mutants being type- 1 (52bp deletion) or type- 2 5 bp insertion) ${ }^{13,14}$ Calreticulin is a highly conserved chaperone protein that directs the correct conformation and intracellular trafficking of glycoproteins, as well as in homeostatic control of calcium levels. Remarkably, calreticulin mutants specifically activate TPOR, and thereby JAK2, after binding to its extracellular $\mathrm{N}$-glycosylation residues. $^{15-17}$

\section{Triple negative MPN}

ET and PMF negative for standard phenotype driver mutations (JAK2, CALR and MPL) are referred to as 'triple negative'. High throughput sequencing identified noncanonical activating $J A K 2$ or MPL mutations in a minority of triple negative MPN, some of which were somatic but others were inherited, thus identifying cases with hereditary thrombocythemia misdiagnosed as MPN. Similarly, some cases had no evidence for a clonal disorder suggesting that they had also been misdiagnosed. Other cases, particularly those with a diagnosis of PMF, had clonal disease associated with a range of mutations characteristic of MDS/MPN and other myeloid disorders e.g. in CBL, SH2B3, TET2, ASXL1 and SRSF2. ${ }^{14,18,19}$

MPN genomes: cytogenetics, arrays and exome sequencing 
Karyotypic abnormalities detected by conventional cytogenetic analysis are seen in a minority of PV and ET (<10\%), but about a third of PMF. ${ }^{20}$ Gain of chromosome 9 is seen recurrently in PV and associated with JAK2 V617F (JAK2 is located at 9p24). Interstitial deletions of $13 q$ and $20 q$ are seen in all MPN subtypes suggesting the presence of one or more tumour suppressor genes in these regions. Of these, 20q deletion is the most frequent and may target the imprinted genes $L 3 M B T L 1$ and SGK2. ${ }^{21}$ Other recurrent abnormalities in MPN for which the molecular basis is unknown include +8 and $\operatorname{der}(6) t(1 ; 6) .^{22}$

Single nucleotide polymorphism (SNP) array analysis has confirmed that PV and ET genomes are relatively simple with very few somatic copy number changes. ${ }^{23,24}$ By contrast, copy number changes are more common in PMF and MF evolving from PV or ET. ${ }^{23,25}$ SNP arrays detect frequent regions of acquired uniparental isodisomy (aUPD) in MPN. Such regions arise from mitotic recombination and enable genetic variants to progress from a heterozygous to a homozygous state without change in DNA copy number. Chromosome $9 p$ is most commonly affected and is almost always associated with homozygosity for JAK2 V617F. ${ }^{6}$ Other chromosomes are affected less frequently, e.g. aUPD at $4 q, 7 q$ and $11 q$ are associated with mutations in TET2, EZH2 and $C B L$, respectively, whereas aUPD14q is associated with an imprinting defect. ${ }^{26-29}$

Exome sequencing identified a median of 6.5 total somatic mutations in ET and PV, increasing to 13 in MF, consistent with the latter being a more advanced stage of disease. ${ }^{14}$ Most of these mutations are passengers that almost certainly play no role in the disease process. RNAseq has not thus far identified any new in-frame oncogenic fusion genes, although this technique may detect loss of function events. ${ }^{30}$ Whole genome sequencing for MPN has not been reported, but may, potentially reveal new somatic changes in non-coding regions of the genome. Overall, however, it is anticipated that the MPN genome is largely understood, at least in terms of the spectrum and frequency of somatic mutations. By contrast, the epigenome remains poorly understood. 
JAK2 V617F can be detected in the general population at a prevalence of $0.1-0.2 \%$, roughly $10 x$ the prevalence of diagnosed MPN. ${ }^{31-35}$ Although some of these mutated individuals turn out to have a previously undiagnosed MPN, many have blood counts within the normal range and thus represent examples of what is now termed age related clonal hematopoiesis $(\mathrm{ARCH})$ or clonal hematopoiesis of indeterminate potential (CHIP). Some of these individuals subsequently develop an MPN but many maintain normal blood counts. A JAK2 V617F mutation burden of $>2 \%$ has been proposed as suggestive of an MPN, ${ }^{36}$ but this is not a clean cut off and some individuals with ARCH /CHIP have higher JAK2 mutation burdens.

Broad population-based mutations screens indicate that ARCH/CHIP is associated with mutations in several different genes with most affected individuals having only one discernible mutation. ${ }^{37-39}$ Up to $50 \%$ of JAK2 V617F mutated PV and ET cases also have no additional pathogenic mutations ${ }^{40,41}$ and thus whilst it appears that JAK2 V617F is sufficient to drive clonal hematopoiesis and an MPN phenotype in many cases, additional factors may play a role in promoting the likelihood of an MPN phenotype developing.

About $50 \%$ of JAK2 V617F mutated ET and PV cases have pathogenic mutations in additional genes, most commonly DNMT3A and TET2. The great majority of JAK2 V617F positive PMF cases have additional mutations, particularly those encoding chromatin modifiers and splicing factors, suggesting that these abnormalities promote an MF phenotype. ${ }^{40,41}$

Remarkably, the order in which mutations are acquired is not fixed. In some patients JAK2 V617F is acquired early in the disease and then additional mutations appear as subsequent subclonal events. In other patients, clinically covert clonal hematopoesis is believed to be established by mutations in DNMT3A, TET2 or other abnormalities such as del(20q) or aUPD14q, and JAK2 V617F is acquired as a later event, triggering the onset of an MPN. The order in which mutations are acquired influences the disease phenotype: patients who acquired JAK2 V617F before TET2, DNMT3A or aUPD14q were more likely to develop PV than ET compared to cases where JAK2 
V617F was acquired late. ${ }^{26,42,43}$ In addition, detailed analysis of TET2/JAK2 comutated patients indicated that that the order in which mutations are acquired influences tumor biology as well as the behaviour of stem and progenitor cells. ${ }^{43}$

In contrast to JAK2 V617F, CALR mutations show a high degree of clonal dominance throughout hematopoietic development and are believed to be the initiating event in most cases. Additional mutations are thus secondary events associated with disease evolution. ${ }^{13,14,40,44}$ The principal genetic abnormalities and evolution of MPN are shown on Figure 2.

\section{Factors determining disease phenotype}

Although MPN phenotype driver mutations all induce the activation of JAK2, they do not lead to the same disorder. JAK2 V617F is associated with PV, ET and PMF while but MPL and CALR mutations are associated with ET and PMF. JAK2 V617F mutant ET patients tend to present many of the phenotypic features of PV and an increased risk of thrombosis compared to ET negative for JAK2 V617F. ${ }^{45,46}$ Cases with MPL mutations are on average older and have higher platelet counts, lower hemoglobin levels and reduced bone marrow cellularity at diagnosis than patients with JAK2 V617F, although, no significant differences in clinical outcome are apparent. ${ }^{47}$

CALR mutations in ET are associated with higher platelet counts, lower haemoglobin levels, lower leukocyte counts and younger age at presentation, while the incidence of thrombotic events is lower than JAK2 V617F positive ET. ${ }^{48-50}$ Although no differences in survival have been demonstrated for the different driver mutation subgroups, ${ }^{50}$ ET patients with type-1 CALR mutations progress to MF much more frequently than those with type-2 mutations. ${ }^{51}$

In PMF the nature of the MPN driver mutation has a stronger prognostic significance. Compared to JAK2 V617F mutated cases, patients with CALR mutations (most commonly type-1 in PMF) present at a younger age, with higher platelet counts and lower leukocyte counts, but with reduced anemia and transfusion dependency, while they have a better prognosis regarding overall and leukemia-free survival. 
Importantly, triple-negative patients for both PMF and post-MPN MF have the worst prognosis and a more severe anemia. ${ }^{48,52,53}$ Compared to PV and ET, the JAK2 V617F and CALR mutant allele burden is significantly higher in MF evolving from either ET or PV, indicating a role for the accumulation of mutated alleles in the process of transformation. ${ }^{48}$

The reason why some JAK2 V617F positive patients present with PV whereas others present with ET or other myeloid disorders is likely to be multifactorial. As discussed above, the JAK2 mutant allele burden is higher in PV compared to ET and the order in which mutations are acquired can influence the disease phenotype. Constitutional genetics also influences the disease phenotype, with inherited variation in the HBS1L-MYB region influencing whether JAK2 V617F mutant cases develop ET or PV. ${ }^{24}$ Phenotypic determinants in MPN may also include factors other than acquired mutations and constitutional genetic modifiers. PV and PMF are more common in males and ET is predominant in females, ${ }^{54}$ whilst CALR-mutated ET is more prevalent in men and JAK2 V617F is more common in women, ${ }^{55}$ suggesting that gender-specific factors may influence MPN phenotype. Hormones could be an explanation for these differences, as oestrogens can inhibit the JAK2 V617F mutated stem cells, ${ }^{56}$ an observation that may explain the observation that JAK2 V617F allele burdens are lower in women compared to men. ${ }^{57}$ Iron status, especially in premenopausal women, is another factor relevant to MPN phenotype. ${ }^{54}$

\section{Disease progression and prognostic significance of disease modifying mutations}

Considerable effort has been put into the identification of genetic predictors of transformation from MPN to AML. Robust molecular prognostic markers are still lacking for patients with PV and ET, and risk stratification for these patients is still based mainly on clinical criteria and the presence of phenotypic driver mutations. The mutational burden is certainly higher on transformation of MPN to AML with the appearance of variants in a range of genes that are not unique to MPN but are also seen in other myeloid malignancies (Table 1). Gene expression profiling with adjustment for gender-specific effects has been suggested to provide prognostic information in $\mathrm{PV},{ }^{58}$ but this approach remains unvalidated. Overall, whilst no strong 
predictors of transformation have been identified, some clinically useful prognostic factors have emerged, particularly for the management of patients with MF.

Mutations in genes involved in transcription or DNA damage response are clearly associated with leukemic transformation, particularly RUNX1 and TP53 but also IKZF1 and CUX1. ${ }^{25,41,59,60}$ Mutations in TP53 may persist in a heterozygous state for an extended period of time during chronic phase MPN without clonal expansion. However, after loss of the wild-type allele by either chromosomal deletion or aUPD, the hemizygous or homozygous TP53 clone rapidly expands, leading to leukemic transformation. ${ }^{41}$ Mutations in RUNX1 and other transcription factors are usually acquired at the time transformation, thus limiting their use as predictive factors. ${ }^{60}$

Impairment of polycomb repressive complex 2 (PRC2) function also appears to be an important pathogenic mechanism that promotes disease progression. Both $E Z H 2$ and ASXL1 mutations are associated with an increased risk of leukemic transformation and inferior survival. ${ }^{41,61-63}$ In PMF, ASXL1 is an important prognostic indicator, with CALR wild type, ASXL1 mutated cases having the worst prognosis. ${ }^{64}$

Mutations in genes encoding chromatin modifiers are not consistently associated with prognosis. Whereas DNMT3A mutations are not considered to be prognostically significant, there are conflicting data regarding the association between TET2 mutations and the risk of leukemic transformation. ${ }^{41,65}$ Rather than promoting disease progression, mutations in these genes may be considered as landscaping events that create a 'fertile ground', i.e. an environment more conducive to the development of a myeloid neoplasm. ${ }^{66}$ Early studies indicated, unexpectedly, that JAK2 V617F mutated MPN may evolve to JAK2 unmutated AML. ${ }^{67,68}$ Most likely this is due to the presence of a pre-existing, clonal phase driven by DNMT3A or TET2 mutations with this clone independently acquiring JAK2 V617F (leading to an MPN) or other mutations (leading to $\mathrm{AML}$ ). Alternatively, it is possible that some individuals have an inherited tendency to develop myeloid malignancies and thus develop MPN and AML as truly independent clones. 
In PMF the presence of mutations in any one of $A S X L 1, S R S F 2, I D H 1 / 2$ or EZH2 has been shown to be associated with reduced overall survival and increased risk for leukemic transformation. ${ }^{69}$ Specifically, the number of these "high-molecular-risk" (HMR) mutations was inversely correlated with median survival in PMF independently of standard risk scores. ${ }^{63}$ Notably, HMR mutations are enriched in triple negative PMF, while spliceosome mutations (SRSF2, SF3B1, U2AF1) are infrequent in CALR-mutant patients compared with the JAK2/MPL-mutant and triplenegative patients. ${ }^{70}$ Mutational analysis in PMF patients who received ruxolitinib revealed that patients with $\geq 3$ mutations of any type had significantly lower odds of a spleen response and a shorter time to treatment discontinuation. Patients with $\geq 1$ mutations in $A S X L 1, E Z H 2, I D H 1$ or $I D H 2$ had shorter survival and time to treatment discontinuation, and were significantly less likely to have a spleen response. ${ }^{71}$ Additional mutations may correlate with other relevant clinical factors, for example in PMF the presence of U2AF1 mutations is strongly associated with severe anemia and thrombocytopenia. ${ }^{72}$ Overall, these data suggest that broad mutational analysis, for PMF patients at least, should be integrated into routine management.

\section{Genetic predisposition}

Familial predisposition to MPN may occur in up to $5-8 \%$ of apparently sporadic cases. ${ }^{73}$ MPN phenotype driver mutations are never inherited but are frequently acquired somatically in familial cases, as they are in sporadic cases. ${ }^{73-75}$ As noted above, inherited, relatively weakly activating JAK2 and MPL mutations are associated with hereditary erythrocytosis or thrombocytosis, disorders that are non-clonal but which may occasionally be mistaken for true MPN. ${ }^{76-78}$

The causes of familial predisposition to MPN are largely unknown. The inheritance patterns are heterogeneous and attempts to aggregate families for linkage analysis have largely failed, suggesting there are probably a variety of different germline mutations of variable penetrance driving the effect. ${ }^{79}$ Thus far, only one clear explanation for high penetrance germline predisposition has emerged: analysis of four large families identified a $14 q$ duplication including ATG2P and GSKIP that segregated with the MPN phenotype (ET/AML) and provided a 'fertile ground' for 
MPN development. ${ }^{80}$ Other abnormalities have also been associated with familial MPN, e.g. germline mutations in the RBBP6 gene in about $5 \%$ of familial cases. ${ }^{81}$

Large epidemiological studies have suggested the presence of weakly penetrant, common risk factors in the general population. ${ }^{82}$ Several of these factors have been identified, but just two (JAK2 46/1 and TERT) account for the major part of the population attributable risk for development of an MPN, although the underlying reasons for this effect remain obscure. JAK2 46/1 (also known as GGCC) strongly predisposes to JAK2-mutated MPN but also, to a much lesser degree, to MPN with other mutations. TERT variants strongly predispose to all MPN. ${ }^{24,31,83-87}$ Although these effects are strong in genetic epidemiological terms, their penetrance is very low and consequently they cannot be used to predict whether any individual is likely to develop an MPN. Nevertheless, aggregation of these factors may contribute to apparent familial clustering. ${ }^{79}$

\section{Non-classic or atypical MPN}

\section{Chronic neutrophilic leukemia}

Frequent oncogenic mutations of CSF3R are seen in CNL, a rare MPN subtype sharing overlapping features with atypical CML. ${ }^{88-90}$ The initial study found that $50-60 \%$ of patients with $\mathrm{CNL}$ or atypical CML harboured CSF3R mutations ${ }^{88}$ with subsequent reports indicating that such abnormalities were restricted to CNL and essentially absent in aCML and MDS/MPN-unclassified..$^{89,90}$ Co-operative mutations in SETBP1 and ASXL1 appear to be of prognostic significance and correlate with disease progression. ${ }^{91}$

CSF3R encodes the receptor for granulocyte-colony stimulating factor 3, which requires JAK2 to function. Mutations fall into 2 types: nonsense or frameshift mutations leading to premature truncation of the cytoplasmic tail of the receptor and point mutations in the extracellular domain, most commonly T618I, an abnormality that strongly activates the JAK/STAT signalling. CSF3R mutations are 
almost always acquired, although a recent report described a child with CNL and an inherited T618I mutation. ${ }^{92}$ Ongoing studies are evaluating whether CNL patients are amenable to treatment with JAK2 or other inhibitors, as suggested by initial reports. $^{88,93}$

\section{Chronic eosinophilic leukemia and MPN-unclassified}

CEL (not otherwise specified) is distinguished from idiopathic hypereosinophilic syndrome largely by the finding of a marker of clonality. Most commonly this has been achieved by cytogenetics, although only a very small proportion of suspected cases turn out to have a karyotypic abnormality. Cytogenetics and/or fluorescent in situ hybridization (FISH) is also critical to help identify cases with FIP1L1-PDGFRA or other tyrosine kinase fusions such as those involving PDGFRA, PDGFRB, FGFR1 or JAK2, abnormalities that are amenable to treatment with specific targeted therapies.

A recent study has demonstrated that targeted next-generation sequencing helps to establish clonality in a subset of patients with cytogenetically normal hypereosinophilia that would otherwise have been classified as idiopathic hypereosinophilic syndrome. Importantly, the survival of these cases was significantly shorter than cases without mutations and was indistinguishable from cases with cytogenetically defined CEL, clearly indicating the value of genomic analysis in this disorder. ${ }^{94}$ There are no molecular markers for MPN-U but it is possible that wide mutation screens may help to better define this heterogeneous condition.

\section{Concluding remarks}

The 2016 revision of WHO classification has incorporated testing for MPN phenotype driver mutations as essential components in the diagnostic workup of MPN. The presence of $B C R-A B L 1$ defines CML but this fusion needs to be excluded in the diagnosis of classic MPN and other tyrosine kinase fusions need to be excluded in 
cases with eosinophilia. It is also suggested that testing for the most frequent disease modifying mutations may assist in the determination of the clonal nature of the disease. ${ }^{1}$ and, as discussed above, many of these abnormalities have prognostic value. Currently, many centers perform a combination of cytogenetics, FISH, reverse transcriptase polymerase chain reaction (RT-PCR), Sanger sequencing and NGS panels in the diagnostic and prognostic work up of MPN. Clearly the time is ripe for genomics-based approaches, for example RNAseq, to supplant these diverse techniques and provide a platform for improved management and understanding of MPN. 


\section{References}

1. Arber DA, Orazi A, Hasserjian R, et al: The 2016 revision to the World Health Organization classification of myeloid neoplasms and acute leukemia. Blood 127:2391-405, 2016

2. Cross NC: Genetic and epigenetic complexity in myeloproliferative neoplasms. Hematology Am Soc Hematol Educ Program 2011:208-14, 2011

3. Vainchenker $W$, Constantinescu SN: JAK/STAT signaling in hematological malignancies. Oncogene 32:2601-13, 2013

4. Baxter EJ, Scott LM, Campbell PJ, et al: Acquired mutation of the tyrosine kinase JAK2 in human myeloproliferative disorders. Lancet 365:1054-61, 2005

5. James C, Ugo V, Le Couedic JP, et al: A unique clonal JAK2 mutation leading to constitutive signalling causes polycythaemia vera. Nature 434:1144-8, 2005

6. Kralovics R, Passamonti F, Buser AS, et al: A gain-of-function mutation of JAK2 in myeloproliferative disorders. N Engl J Med 352:1779-90, 2005

7. Levine $\mathrm{RL}$, Loriaux $\mathrm{M}$, Huntly $\mathrm{BJ}$, et al: The JAK2V617F activating mutation occurs in chronic myelomonocytic leukemia and acute myeloid leukemia, but not in acute lymphoblastic leukemia or chronic lymphocytic leukemia. Blood 106:3377-9, 2005

8. Godfrey AL, Nangalia J, Baxter EJ, et al: Nongenetic stochastic expansion of JAK2V617F-homozygous subclones in polycythemia vera? Blood $124: 3332-4,2014$

9. Scott $\mathrm{LM}$, Tong $\mathrm{W}$, Levine $\mathrm{RL}$, et al: JAK2 exon 12 mutations in polycythemia vera and idiopathic erythrocytosis. N Engl J Med 356:459-68, 2007

10. Defour JP, Chachoua I, Pecquet C, et al: Oncogenic activation of $\mathrm{MPL} /$ thrombopoietin receptor by 17 mutations at W515: implications for myeloproliferative neoplasms. Leukemia 30:1214-6, 2016

11. Pikman $Y$, Lee $B H$, Mercher $T$, et al: MPLW515L is a novel somatic activating mutation in myelofibrosis with myeloid metaplasia. PLoS Med 3:e270, 2006

12. Defour JP, Itaya $M$, Gryshkova $V$, et al: Tryptophan at the transmembrane-cytosolic junction modulates thrombopoietin receptor dimerization and activation. Proc Natl Acad Sci U S A 110:2540-5, 2013

13. Klampfl T, Gisslinger $H$, Harutyunyan AS, et al: Somatic mutations of calreticulin in myeloproliferative neoplasms. N Engl J Med 369:2379-90, 2013

14. Nangalia J, Massie CE, Baxter EJ, et al: Somatic CALR mutations in myeloproliferative neoplasms with nonmutated JAK2. N Engl J Med 369:2391-405, 2013

15. Chachoua I, Pecquet C, El-Khoury $M$, et al: Thrombopoietin receptor activation by myeloproliferative neoplasm associated calreticulin mutants. Blood 127:1325-35, 2016

16. Elf S, Abdelfattah NS, Chen E, et al: Mutant Calreticulin Requires Both Its Mutant C-terminus and the Thrombopoietin Receptor for Oncogenic Transformation. Cancer Discov 6:368-81, 2016 
17. Marty $\mathrm{C}$, Pecquet $\mathrm{C}$, Nivarthi $\mathrm{H}$, et al: Calreticulin mutants in mice induce an MPL-dependent thrombocytosis with frequent progression to myelofibrosis. Blood 127:1317-24, 2016

18. Cabagnols $\mathrm{X}$, Favale $\mathrm{F}$, Pasquier $\mathrm{F}$, et al: Presence of atypical thrombopoietin receptor (MPL) mutations in triple-negative essential thrombocythemia patients. Blood 127:333-42, 2016

19. Milosevic Feenstra JD, Nivarthi $H$, Gisslinger $H$, et al: Whole-exome sequencing identifies novel MPL and JAK2 mutations in triple-negative myeloproliferative neoplasms. Blood 127:325-32, 2016

20. Bench AJ, White HE, Foroni L, et al: Molecular diagnosis of the myeloproliferative neoplasms: UK guidelines for the detection of JAK2 V617F and other relevant mutations. Br J Haematol 160:25-34, 2013

21. Aziz A, Baxter EJ, Edwards C, et al: Cooperativity of imprinted genes inactivated by acquired chromosome 20q deletions. J Clin Invest 123:2169-82, 2013

22. Dingli $D$, Grand FH, Mahaffey V, et al: $\operatorname{Der}(6) t(1 ; 6)(q 21-23 ; p 21.3)$ : a specific cytogenetic abnormality in myelofibrosis with myeloid metaplasia. $\mathrm{Br} \mathrm{J}$ Haematol 130:229-32, 2005

23. Stegelmann F, Bullinger L, Griesshammer $\mathrm{M}$, et al: High-resolution single-nucleotide polymorphism array-profiling in myeloproliferative neoplasms identifies novel genomic aberrations. Haematologica 95:666-9, 2010

24. Tapper W, Jones AV, Kralovics $R$, et al: Genetic variation at MECOM, TERT, JAK2 and HBS1L-MYB predisposes to myeloproliferative neoplasms. Nat Commun 6:6691, 2015

25. Klampfl T, Harutyunyan A, Berg $T$, et al: Genome integrity of myeloproliferative neoplasms in chronic phase and during disease progression. Blood 118:167-76, 2011

26. Chase A, Leung W, Tapper W, et al: Profound parental bias associated with chromosome 14 acquired uniparental disomy indicates targeting of an imprinted locus. Leukemia 29:2069-74, 2015

27. Delhommeau F, Dupont S, Della Valle V, et al: Mutation in TET2 in myeloid cancers. N Engl J Med 360:2289-301, 2009

28. Ernst T, Chase AJ, Score J, et al: Inactivating mutations of the histone methyltransferase gene EZH2 in myeloid disorders. Nat Genet 42:722-6, 2010

29. Grand $\mathrm{FH}$, Hidalgo-Curtis $\mathrm{CE}$, Ernst $\mathrm{T}$, et al: Frequent $\mathrm{CBL}$ mutations associated with $11 \mathrm{q}$ acquired uniparental disomy in myeloproliferative neoplasms. Blood 113:6182-92, 2009

30. Schischlik F, Milosevic Feenstra, J.D., Rumi, E.,Pietra, D., Gisslinger,B., Schalling, M., Bogner, E., Gisslinger, H., Cazzola, M., Kralovics, R.: Fusion Gene Detection Using Whole Transcriptome Analysis in Patients with Chronic Myeloproliferative Neoplasms and Secondary Acute Myeloid Leukemia. Blood 126:4093, 2015

31. Hinds DA, Barnholt KE, Mesa RA, et al: Germ line variants predispose to both JAK2 V617F clonal hematopoiesis and myeloproliferative neoplasms. Blood 128:1121-8, 2016

32. McKerrell T, Park N, Moreno T, et al: Leukemia-associated somatic mutations drive distinct patterns of age-related clonal hemopoiesis. Cell Rep 10:1239-45, 2015 
33. Nielsen C, Birgens HS, Nordestgaard BG, et al: The JAK2 V617F somatic mutation, mortality and cancer risk in the general population. Haematologica 96:450-3, 2011

34. Roaldsnes $\mathrm{C}$, Holst $\mathrm{R}$, Frederiksen $\mathrm{H}$, et al: Myeloproliferative neoplasms: trends in incidence, prevalence and survival in Norway. Eur J Haematol, 2016

35. Xu X, Zhang Q, Luo J, et al: JAK2(V617F): Prevalence in a large Chinese hospital population. Blood 109:339-42, 2007

36. Nielsen C, Bojesen SE, Nordestgaard BG, et al: JAK2V617F somatic mutation in the general population: myeloproliferative neoplasm development and progression rate. Haematologica 99:1448-55, 2014

37. Busque L, Patel JP, Figueroa ME, et al: Recurrent somatic TET2 mutations in normal elderly individuals with clonal hematopoiesis. Nat Genet 44:1179-81, 2012

38. Genovese G, Kahler AK, Handsaker RE, et al: Clonal hematopoiesis and blood-cancer risk inferred from blood DNA sequence. N Engl J Med 371:2477-87, 2014

39. Jaiswal S, Fontanillas $\mathrm{P}$, Flannick J, et al: Age-related clonal hematopoiesis associated with adverse outcomes. N Engl J Med 371:2488-98, 2014

40. Delic S, Rose D, Kern W, et al: Application of an NGS-based 28-gene panel in myeloproliferative neoplasms reveals distinct mutation patterns in essential thrombocythaemia, primary myelofibrosis and polycythaemia vera. $\mathrm{Br} \mathrm{J}$ Haematol, 2016

41. Lundberg $P$, Karow A, Nienhold R, et al: Clonal evolution and clinical correlates of somatic mutations in myeloproliferative neoplasms. Blood 123:2220-8, 2014

42. Nangalia J, Nice FL, Wedge DC, et al: DNMT3A mutations occur early or late in patients with myeloproliferative neoplasms and mutation order influences phenotype. Haematologica 100:e438-42, 2015

43. Ortmann CA, Kent DG, Nangalia J, et al: Effect of mutation order on myeloproliferative neoplasms. N Engl J Med 372:601-12, 2015

44. Lundberg $P$, Nienhold $R$, Ambrosetti $A$, et al: Somatic mutations in calreticulin can be found in pedigrees with familial predisposition to myeloproliferative neoplasms. Blood 123:2744-5, 2014

45. Antonioli $E$, Guglielmelli $P$, Pancrazzi A, et al: Clinical implications of the JAK2 V617F mutation in essential thrombocythemia. Leukemia 19:1847-9, 2005

46. Campbell PJ, Scott LM, Buck G, et al: Definition of subtypes of essential thrombocythaemia and relation to polycythaemia vera based on JAK2 V617F mutation status: a prospective study. Lancet 366:1945-53, 2005

47. Vannucchi AM, Antonioli E, Guglielmelli $P$, et al: Characteristics and clinical correlates of MPL $515 \mathrm{~W}>\mathrm{L} / \mathrm{K}$ mutation in essential thrombocythemia. Blood 112:844-7, 2008

48. Rotunno G, Pacilli A, Artusi V, et al: Epidemiology and clinical relevance of mutations in postpolycythemia vera and postessential thrombocythemia myelofibrosis: A study on 359 patients of the AGIMM group. Am J Hematol 91:681-6, 2016 
49. Rumi E, Pietra D, Ferretti V, et al: JAK2 or CALR mutation status defines subtypes of essential thrombocythemia with substantially different clinical course and outcomes. Blood 123:1544-51, 2014

50. Tefferi A, Guglielmelli P, Larson DR, et al: Long-term survival and blast transformation in molecularly annotated essential thrombocythemia, polycythemia vera, and myelofibrosis. Blood 124:2507-13; quiz 2615, 2014

51. Pietra D, Rumi E, Ferretti VV, et al: Differential clinical effects of different mutation subtypes in CALR-mutant myeloproliferative neoplasms. Leukemia 30:431-8, 2016

52. Rumi E, Pietra D, Pascutto $C$, et al: Clinical effect of driver mutations of JAK2, CALR, or MPL in primary myelofibrosis. Blood 124:1062-9, 2014

53. Tefferi A, Lasho TL, Finke CM, et al: CALR vs JAK2 vs MPL-mutated or triple-negative myelofibrosis: clinical, cytogenetic and molecular comparisons. Leukemia 28:1472-7, 2014

54. Godfrey AL, Chen E, Pagano F, et al: Clonal analyses reveal associations of JAK2V617F homozygosity with hematologic features, age and gender in polycythemia vera and essential thrombocythemia. Haematologica 98:718-21, 2013

55. Cabagnols $X$, Defour JP, Ugo $V$, et al: Differential association of calreticulin type 1 and type 2 mutations with myelofibrosis and essential thrombocytemia: relevance for disease evolution. Leukemia 29:249-52, 2015

56. Sanchez-Aguilera A, Arranz L, Martin-Perez D, et al: Estrogen signaling selectively induces apoptosis of hematopoietic progenitors and myeloid neoplasms without harming steady-state hematopoiesis. Cell Stem Cell 15:791-804, 2014

57. Stein BL, Williams DM, Wang NY, et al: Sex differences in the JAK2 V617F allele burden in chronic myeloproliferative disorders. Haematologica 95:10907, 2010

58. Spivak JL, Considine M, Williams DM, et al: Two clinical phenotypes in polycythemia vera. N Engl J Med 371:808-17, 2014

59. Cerquozzi S, Tefferi A: Blast transformation and fibrotic progression in polycythemia vera and essential thrombocythemia: a literature review of incidence and risk factors. Blood Cancer J 5:e366, 2015

60. Milosevic JD, Puda A, Malcovati L, et al: Clinical significance of genetic aberrations in secondary acute myeloid leukemia. Am J Hematol 87:1010-6, 2012

61. Brecqueville $M$, Rey J, Bertucci $F$, et al: Mutation analysis of ASXL1, CBL, DNMT3A, IDH1, IDH2, JAK2, MPL, NF1, SF3B1, SUZ12, and TET2 in myeloproliferative neoplasms. Genes Chromosomes Cancer 51:743-55, 2012

62. Guglielmelli P, Biamonte F, Score J, et al: EZH2 mutational status predicts poor survival in myelofibrosis. Blood 118:5227-34, 2011

63. Guglielmelli $P$, Lasho $T L$, Rotunno $G$, et al: The number of prognostically detrimental mutations and prognosis in primary myelofibrosis: an international study of 797 patients. Leukemia, 2014

64. Tefferi A, Guglielmelli P, Lasho TL, et al: CALR and ASXL1 mutationsbased molecular prognostication in primary myelofibrosis: an international study of 570 patients. Leukemia 28:1494-500, 2014 
65. Tefferi A, Pardanani A, Lim KH, et al: TET2 mutations and their clinical correlates in polycythemia vera, essential thrombocythemia and myelofibrosis. Leukemia 23:905-11, 2009

66. Corces-Zimmerman MR, Hong WJ, Weissman IL, et al: Preleukemic mutations in human acute myeloid leukemia affect epigenetic regulators and persist in remission. Proc Natl Acad Sci U S A 111:2548-53, 2014

67. Campbell PJ, Baxter EJ, Beer PA, et al: Mutation of JAK2 in the myeloproliferative disorders: timing, clonality studies, cytogenetic associations, and role in leukemic transformation. Blood 108:3548-55, 2006

68. Theocharides A, Boissinot $M$, Girodon $F$, et al: Leukemic blasts in transformed JAK2-V617F-positive myeloproliferative disorders are frequently negative for the JAK2-V617F mutation. Blood 110:375-9, 2007

69. Vannucchi AM, Lasho TL, Guglielmelli $P$, et al: Mutations and prognosis in primary myelofibrosis. Leukemia 27:1861-9, 2013

70. Guglielmelli P, Rotunno, G., Fanelli, T., Pacilli, A., Corbizi-Fattori, G., Calabresi, L., Pancrazzi, A., Vannucchi, A.M.: A greater underlying mutational complexity may contribute to the differential prognostic impact of phenotypic-driver mutation in primary myelofibrosis. EHA21 Congress. Copenhagen, Haematologica, 2016, pp 8-9

71. Patel KP, Newberry KJ, Luthra R, et al: Correlation of mutation profile and response in patients with myelofibrosis treated with ruxolitinib. Blood 126:7907, 2015

72. Tefferi A, Finke CM, Lasho TL, et al: U2AF1 mutations in primary myelofibrosis are strongly associated with anemia and thrombocytopenia despite clustering with JAK2V617F and normal karyotype. Leukemia 28:431-3, 2014

73. Rumi E, Passamonti F, Della Porta MG, et al: Familial chronic myeloproliferative disorders: clinical phenotype and evidence of disease anticipation. J Clin Oncol 25:5630-5, 2007

74. Bellanne-Chantelot C, Chaumarel I, Labopin M, et al: Genetic and clinical implications of the Val617Phe JAK2 mutation in 72 families with myeloproliferative disorders. Blood 108:346-52, 2006

75. Rumi E, Harutyunyan AS, Pietra D, et al: CALR exon 9 mutations are somatically acquired events in familial cases of essential thrombocythemia or primary myelofibrosis. Blood 123:2416-9, 2014

76. Ding J, Komatsu $H$, Wakita $A$, et al: Familial essential thrombocythemia associated with a dominant-positive activating mutation of the cMPL gene, which encodes for the receptor for thrombopoietin. Blood 103:4198-200, 2004

77. Kapralova K, Horvathova M, Pecquet C, et al: Cooperation of germ line JAK2 mutations E846D and R1063H in hereditary erythrocytosis with megakaryocytic atypia. Blood 128:1418-23, 2016

78. Mead AJ, Chowdhury O, Pecquet $C$, et al: Impact of isolated germline JAK2V617I mutation on human hematopoiesis. Blood 121:4156-65, 2013

79. Jager R, Harutyunyan AS, Rumi $E$, et al: Common germline variation at the TERT locus contributes to familial clustering of myeloproliferative neoplasms. Am J Hematol 89:1107-10, 2014 
80. Saliba J, Saint-Martin C, Di Stefano A, et al: Germline duplication of ATG2B and GSKIP predisposes to familial myeloid malignancies. Nat Genet 47:113140, 2015

81. Harutyunyan AS, Giambruno R, Krendl C, et al: Germline RBBP6 mutations in familial myeloproliferative neoplasms. Blood 127:362-5, 2016

82. Landgren $\mathrm{O}$, Goldin LR, Kristinsson SY, et al: Increased risks of polycythemia vera, essential thrombocythemia, and myelofibrosis among 24,577 first-degree relatives of 11,039 patients with myeloproliferative neoplasms in Sweden. Blood 112:2199-204, 2008

83. Jones AV, Campbell PJ, Beer PA, et al: The JAK2 46/1 haplotype predisposes to MPL-mutated myeloproliferative neoplasms. Blood 115:4517-23, 2010

84. Jones $\mathrm{AV}$, Chase $\mathrm{A}$, Silver RT, et al: JAK2 haplotype is a major risk factor for the development of myeloproliferative neoplasms. Nat Genet 41:446-9, 2009

85. Kilpivaara O, Mukherjee S, Schram AM, et al: A germline JAK2 SNP is associated with predisposition to the development of JAK2(V617F)-positive myeloproliferative neoplasms. Nat Genet 41:455-9, 2009

86. Oddsson A, Kristinsson SY, Helgason $\mathrm{H}$, et al: The germline sequence variant rs2736100_C in TERT associates with myeloproliferative neoplasms. Leukemia 28:1371-4, 2014

87. Olcaydu D, Harutyunyan A, Jager $R$, et al: A common JAK2 haplotype confers susceptibility to myeloproliferative neoplasms. Nat Genet 41:450-4, 2009

88. Maxson JE, Gotlib J, Pollyea DA, et al: Oncogenic CSF3R mutations in chronic neutrophilic leukemia and atypical CML. N Engl J Med 368:1781-90, 2013

89. Pardanani A, Lasho TL, Laborde RR, et al: CSF3R T618I is a highly prevalent and specific mutation in chronic neutrophilic leukemia. Leukemia 27:18703, 2013

90. Wang SA, Hasserjian RP, Fox PS, et al: Atypical chronic myeloid leukemia is clinically distinct from unclassifiable myelodysplastic/myeloproliferative neoplasms. Blood 123:2645-51, 2014

91. Elliott MA, Tefferi A: Chronic neutrophilic leukemia 2016: Update on diagnosis, molecular genetics, prognosis, and management. Am J Hematol 91:341-9, 2016

92. Druhan $\amalg$, McMahon DP, Steuerwald N, et al: Chronic neutrophilic leukemia in a child with a CSF3R T618I germline mutation. Blood, 2016

93. Gotlib J, Maxson JE, George TI, et al: The new genetics of chronic neutrophilic leukemia and atypical CML: implications for diagnosis and treatment. Blood 122:1707-11, 2013

94. Wang SA, Tam W, Tsai AG, et al: Targeted next-generation sequencing identifies a subset of idiopathic hypereosinophilic syndrome with features similar to chronic eosinophilic leukemia, not otherwise specified. Mod Pathol 29:854-64, 2016

95. Lasho TL, Pardanani A, Tefferi A: LNK mutations in JAK2 mutationnegative erythrocytosis. N Engl J Med 363:1189-90, 2010

96. Tefferi A, Lasho TL, Abdel-Wahab O, et al: IDH1 and IDH2 mutation studies in 1473 patients with chronic-, fibrotic- or blast-phase essential thrombocythemia, polycythemia vera or myelofibrosis. Leukemia 24:1302-9, 2010 
97. Oh ST, Simonds EF, Jones $\mathrm{C}$, et al: Novel mutations in the inhibitory adaptor protein LNK drive JAK-STAT signaling in patients with myeloproliferative neoplasms. Blood 116:988-92, 2010

98. Score J, Hidalgo-Curtis C, Jones AV, et al: Inactivation of polycomb repressive complex 2 components in myeloproliferative and myelodysplastic/myeloproliferative neoplasms. Blood 119:1208-13, 2012 
Table 1. Approximate frequencies of acquired mutations in MPNs

\begin{tabular}{|c|c|c|c|c|c|c|c|c|}
\hline \multirow[b]{2}{*}{ Gene } & \multirow[b]{2}{*}{$\begin{array}{l}\text { Genomic } \\
\text { location }\end{array}$} & \multirow[b]{2}{*}{$\begin{array}{l}\text { Mutation } \\
\text { location }\end{array}$} & \multirow[b]{2}{*}{ Protein } & \multirow[b]{2}{*}{ Mutation effect } & \multicolumn{4}{|c|}{ Mutation frequency in MPN, \% of cases } \\
\hline & & & & & PV & ET & PMF & $\begin{array}{l}\text { Blast } \\
\text { phase }\end{array}$ \\
\hline \multicolumn{9}{|c|}{ Phenotypic/Driver Mutations } \\
\hline JAK2 & $9 q 24$ & exon 14 & JAK2 & JAK/STAT signalling & $95-97$ & 60 & 60 & 50 \\
\hline JAK2 & $9 q 24$ & exon 12 & JAK2 & JAK/STAT signalling & $1-2$ & 0 & Rare & $\mathrm{nk}$ \\
\hline MPL & $1 q 34$ & exon 10 & TPOR & JAK/STAT signalling & 0 & $3-5$ & $5-10$ & nk \\
\hline CALR & $19 q 13$ & exon 9 & CALR & JAK/STAT signalling & 0 & 25 & 30 & nk \\
\hline \multicolumn{9}{|c|}{ Initiation/Landscaping mutations } \\
\hline TET2 & $4 q 24$ & $\begin{array}{l}\text { all coding } \\
\text { regions }\end{array}$ & TET2 & Epigenetic regulation & $10-20$ & 5 & $10-20$ & 20 \\
\hline DNMT3A & $2 p 23$ & exons $7-23$ & DNMT3A & Epigenetic regulation & $5-10$ & $1-5$ & $8-12$ & $10-20$ \\
\hline \multicolumn{9}{|c|}{ Disease Modifying/Progression Mutations } \\
\hline IDH1/2 & $2 q 33 / 15 q 26$ & $\begin{array}{l}\text { exon } 4 \text { exon } 4 \\
\text { all coding }\end{array}$ & IDH1/2 & Epigenetic regulation & $1-2$ & $1-2$ & $5-6$ & $10-30$ \\
\hline EZH2 & $7 q 35-q 36$ & regions & $\mathrm{EZH} 2$ & Epigenetic regulation & $1-2$ & $1-2$ & $7-10$ & 5 \\
\hline ASXL1 & $20 q 11$ & exon 12 & ASXL1 & Epigenetic regulation & 2 & $2-5$ & $10-35$ & $20-25$ \\
\hline SF3B1 & $2 q 33$ & exons $12-16$ & SF3B1 & mRNA splicing & 2 & 2 & 5 & 5 \\
\hline SRSF2 & $17 q 25$ & exon 1 & SRSF2 & mRNA splicing & Rare & Rare & $5-17$ & $15-25$ \\
\hline U2AF1 & $21 q 22$ & exons $2-7$ & U2AF1 & mRNA splicing & Rare & Rare & $2-8$ & nk \\
\hline ZRSR2 & Xp22 & $\begin{array}{l}\text { all coding } \\
\text { regions } \\
\text { all coding }\end{array}$ & ZRSR2 & mRNA splicing & Rare & Rare & 1 & nk \\
\hline RUNX1 & $21 q 22$ & regions & RUNX1 & Transcription & nk & nk & nk & $10-15$ \\
\hline
\end{tabular}




\begin{tabular}{|c|c|c|c|c|c|c|c|c|}
\hline IKZF1 & $7 p 12$ & $\begin{array}{l}\text { all coding } \\
\text { regions }\end{array}$ & Ikaros & Transcription & Rare & Rare & Rare & 20 \\
\hline TP53 & 17p13 & $\begin{array}{l}\text { all coding } \\
\text { regions } \\
\text { all coding }\end{array}$ & TP53 & DNA repair & Rare & Rare & Rare & 25 \\
\hline CUX1 & $7 q 22$ & regions & CUX1 & Cell cycle & $\mathrm{nk}$ & $\mathrm{nk}$ & nk & 5 \\
\hline$C B L$ & $11 q 23$ & exons 8-9 & $\mathrm{CBL}$ & JAK/STAT signalling & Rare & Rare & $5-10$ & $6-9$ \\
\hline SH2B3 & $12 q 24$ & exon 2 & LNK & JAK/STAT signalling & $1-2$ & $3-6$ & $3-6$ & 10 \\
\hline
\end{tabular}


Figure legends

Figure 1. Phenotypic driver mutations in classic, $B C R-A B L 1$ negative MPN

Figure 2. Pathways and principal genetic abnormalities in the evolution of $B C R-A B L 1$ negative MPN 


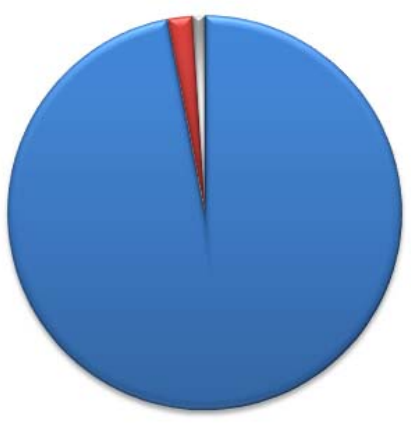

PV

97\% JAK2 V617F

1\% JAK2 exon 12

- $2 \%$ UNKNOWN

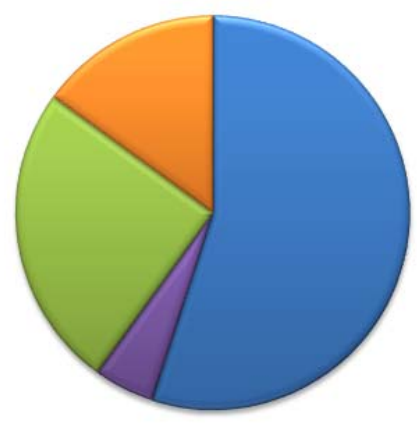

ET

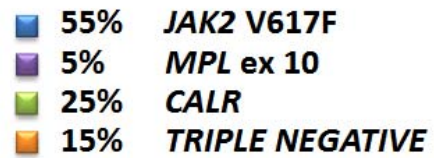

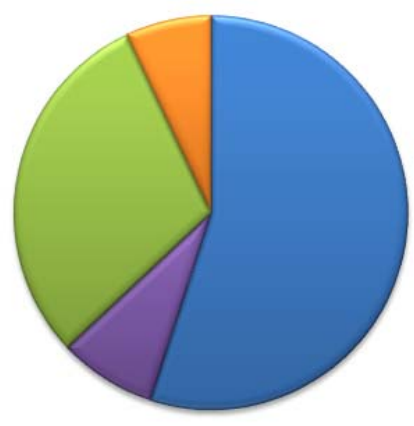

PMF

$\begin{array}{lll}55 \% & \text { JAK2 V617F } \\ 8 \% & \text { MPL ex } 10 \\ 30 \% & \text { CALR } \\ 7 \% & \text { TRIPLE NEGATIVE }\end{array}$

Figure 1

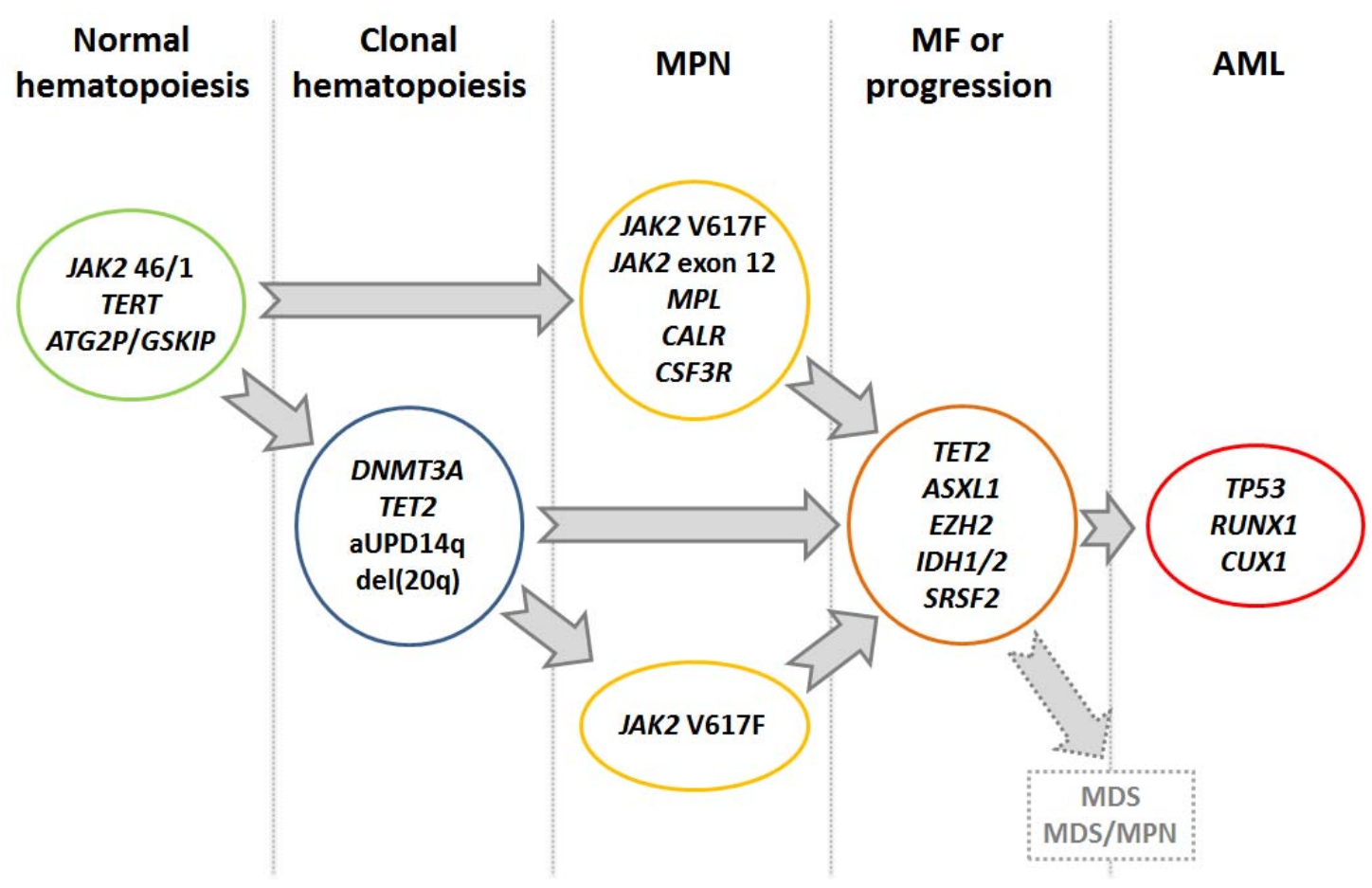

Figure 2 